\title{
ESTROGEN AND AGING AFFECT SYNAPTIC DISTRIBUTION OF PHOSPHORYLATED LIM KINASE (pLIMK) IN CA1 REGION OF FEMALE RAT HIPPOCAMPUS
}

\author{
M. YILDIRIM, ${ }^{a, b, c}$ W. G. M. JANSSEN, ${ }^{b}$ N. E. TABORI, ${ }^{c, d}$

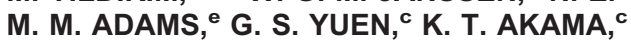 \\ B. S. McEWEN, ${ }^{c}$ T. A. MILNER ${ }^{c, d}$ AND J. H. MORRISON ${ }^{b *}$ \\ ${ }^{a}$ Department of Pharmacology, Hacettepe University, Faculty of Med- \\ icine, Ankara, Turkey, 06100 \\ ${ }^{b}$ Fishberg Department of Neuroscience and Kastor Neurobiology of \\ Aging Laboratories, Box 1639, One Gustave L. Levy Place, Mount \\ Sinai School of Medicine, New York, NY 10029, USA \\ 'Laboratory of Neuroendocrinology, Rockefeller University, New York, \\ NY 10021, USA \\ ${ }^{d}$ Weill-Cornell Medical College, Department of Neurology and Neuro- \\ science, New York, NY 10021, USA \\ ${ }^{e}$ Department of Neurobiology and Anatomy, Wake Forest University \\ School of Medicine, Winston-Salem, NC 27157, USA
}

\begin{abstract}
Estradiol (E) increases axospinous synapse density in the hippocampal CA1 region of young female rats, but not in aged rats. This may be linked to age-related alterations in signaling pathways activated by synaptic estrogen receptor $\alpha(E R-\alpha)$ that potentially regulate spine formation, such as LIM-kinase (LIMK), an actin depolymerizing factor/ cofilin kinase. We hypothesized that, as with ER- $\alpha$, phosphoLIM-kinase (pLIMK) may be less abundant or responsive to $E$ in CA1 synapses of aged female rats. To address this, cellular and subcellular distribution of pLIMK-immunoreactivity (IR) in CA1 was analyzed by light and electron microscopy in young and aged female rats that were ovariectomized and treated with either vehicle or E. pLIMK-IR was found primarily in perikarya within the pyramidal cell layer and dendritic shafts and spines in stratum radiatum (SR). While pLIMK-IR was occasionally present in terminals, post-embedding quantitative analysis of SR showed that pLIMK had a predominant post-synaptic localization and was preferentially localized within the postsynaptic density (PSD). The percentage of pLIMK-labeled synapses increased $(30 \%)$ with $E$ treatment $(P<0.02)$ in young animals, and decreased $(43 \%)$ with age $(P<0.002)$ regardless of treatment. The pattern of distribution of pLIMK-IR within dendritic spines and synapses was unaffected by age or E treatment, with the exception of an E-induced increase in the non-synaptic core of spines in young females. These data suggest that age-related synaptic alterations similar to those seen with ER- $\alpha$ occur with signaling molecules such as PLIMK, and support the hypothesis that age-related failure of $E$ treatment to increase synapse number in CA1 may be due to changes in the molecular profile of axospinous synapses with respect to sig-
\end{abstract}

${ }^{*}$ Corresponding author. Tel: +1-212-659-5985; fax: +1-212-849-2510. E-mail address: john.morrison@mssm.edu (J. H. Morrison).

Abbreviations: BSA, bovine serum albumin; E, $17 \beta$-estradiol; EM, electron microscopy; EMS, Electron Microscopy Sciences; ER, estrogen receptor; HSA, human serum albumin; IR, immunoreactivity/immunoreactive; LIMK, LIM-kinase; LM, light microscopy; LTD, longterm depression; LTP, long-term potentiation; OVX, ovariectomy; PB, phosphate buffer; pLIMK, phosphorylated-LIM kinase; PSD, postsynaptic density; TS, Tris-saline; Veh, vehicle. naling pathways linked to formation of additional spines and synapses in response to E. (C) 2008 IBRO. Published by Elsevier Ltd. All rights reserved.

Key words: sex steroids, electron microscopy, immunogold, signal transduction, plasticity, synapse.

In the female rat hippocampus, dendritic spine density and synapse number in CA1 pyramidal neurons increase when levels of estrogen increase (Gould et al., 1990; Woolley and McEwen, 1992; Woolley et al., 1996), and the size of CA1 spines is also affected by estrogen (Mukai et al., 2007). Estrogen receptor (ER) $-\alpha$ and ER- $\beta$ are both present in these same spines and synapses (Milner et al., 2001, 2005; Adams et al., 2002) and while ER- $\beta$ also impacts synaptic plasticity in CA1 (Day et al., 2005; Szymczak et al., 2006), the estrogen-induced spinogenesis in CA1 is thought to be mediated primarily by ER- $\alpha$ (Mukai et al., 2006, 2007; Jelks et al., 2007). Estrogen fails to increase CA1 spine/synapse density in aged female rats (Adams et al., 2001c), which may be due to decreased ER- $\alpha$ in CA1 synapses of aged rats (Adams et al., 2002). This suggests that signaling molecules involved in the dynamic regulation of spine morphology may be linked to estrogen through activation of ER- $\alpha$ and that such molecules may also be present in these synapses and decreased with age.

One such target molecule of interest is phosphorylatedLIM kinase (pLIMK), a serine/threonine kinase (Okano et al., 1995) that is activated by estrogen in young female hippocampus (Yuen et al., 2004) and plays a key role in the dynamic regulation of actin polymerization and depolymerization through phosphorylation of cofilin (Arber et al., 1998; Yang et al., 1998). Actin impacts synaptic plasticity through its critical role in spine formation, regulation of spine size, and linkage to the synapse (Zhou et al., 2001; Fukazawa et al., 2003; Okamoto et al., 2004; Carlisle and Kennedy, 2005). Cofilin, a member of the actin-depolymerizing factor (ADF)/cofilin family, is a key actin binding protein that promotes turnover and reassembly of actin in living cells (Carlier et al., 1997; Rosenblatt et al., 1997; Bamburg, 1999), and cofilin's role in actin depolymerization is particularly important in neurons (Sarmiere and Bamburg, 2004). Recently, it was demonstrated that cofilin is present in CA1 spines and enriched near the membrane and in the postsynaptic density (PSD), ideally positioning it for regulation of actin dynamics in the context of synaptic activity (Racz and Weinberg, 2006), and such linkages between cofilin, long-term depression (LTD), long-term po- 
tentiation (LTP) and spine size have been demonstrated (Zhou et al., 2004; Fedulov et al., 2007).

While cofilin directly binds to actin and promotes its reassembly, pLIMK is a key regulator of cofilin activity, in that it inactivates cofilin through phosphorylation (Arber et al., 1998; Yang et al., 1998; Maekawa et al., 1999; Ohashi et al., 2000b), inhibiting cofilin's capacity to bind to and depolymerize actin (Meberg, 2000; Ohashi et al., 2000a; Endo et al., 2003; Foletta et al., 2003; Meng et al., 2004; Vardouli et al., 2005). The phosphorylation of cofilin by pLIMK is opposed by slingshot, a phosphatase that removes the phosphate group added by PLIMK (Endo et al., 2003; Wang et al., 2005), suggesting that the cofilin/ actin interaction affecting spine morphology and other neuronal morphologic attributes is highly regulated by the interplay of pLIMK and slingshot (Wen et al., 2007). In fact, disruption of pLIMK leads to alterations in both spine morphology and synaptic properties (Meng et al., 2002, 2003), presumably through disrupted control of cofilin's depolymerization of actin.

Such a signaling cascade provides pLIMK with the potential to play a key role in regulation of spine formation in general, and potentially in estrogen-induced spinogenesis in particular. However, to participate in the regulation of spinogenesis in CA1 pyramidal cells through this mechanism, pLIMK must be present in CA1 spines and synapses with a distribution allowing for activation by ER- $\alpha$ and phosphorylation of cofilin. In addition, if this signaling cascade is compromised in aging leading to a blunted ability of estrogen to promote spinogenesis, then synaptic distribution of pLIMK should be altered in aged female rats. We performed a detailed analysis of pLIMK distribution in CA1 of young and aged ovariectomized female rats with and without estrogen treatment to test these predictions.

\section{EXPERIMENTAL PROCEDURES}

\section{Light microscopy (LM) and pre-embedding electron microscopy (EM)}

Animals. Female Sprague-Dawley rats $(N=6 ; 275-300 \mathrm{~g}$; Taconic Farms, Chatham, NY, USA) were used in the light microscopic and pre-embedding electron microscopic analysis. Animals were housed in a temperature-controlled room (12-h light/dark cycle; lights on at 07:00 h). Food and water were available ad libitum. All experiments were conducted in accordance with Guidelines for the Care and Use of Experimental Animals, by using protocols approved by the Institutional Animal Care and Use Committees at Weill-Cornell Medical College and Rockefeller University. All efforts were made to minimize the number of animals used and their suffering.

Anti-pLIMK antibody. The anti-pLIMK antibody (Cell Signaling Technologies, Beverly, MA, USA), which was used in this study, has been well characterized (Okano et al., 1995). It is a polyclonal antibody generated in the rabbit with a synthetic phospho-peptide (KLH coupled) corresponding to residues surrounding Thr508 of human LIM-kinase (LIMK). The antibody was purified by protein $A$ and peptide affinity chromatography. The specificity of this antibody has been demonstrated extensively in Western blots and tissue staining (Okano et al., 1995; Maekawa et al., 1999; Ohashi et al., 2000b). This antibody recognizes pLIMK-1 and pLIMK-2, when phosphorylated at threonine 508 or
505, respectively (Edwards et al., 1999; Ohashi et al., 2000b; Sumi et al., 2001). All the experiments and measurements were conducted for total pLIMK, and all results were given for total pLIMK distribution.

Immunocytochemistry. Rats were deeply anesthetized with sodium pentobarbital $(150 \mathrm{mg} / \mathrm{kg}$, i.p.) and perfused through the ascending aorta sequentially with solutions of: 1) $10-15 \mathrm{ml}$ saline $(0.9 \%)$ containing 1000 units of heparin; 2) $50 \mathrm{ml}$ of $3.75 \%$ acrolein and $2 \%$ paraformaldehyde in $0.1 \mathrm{M}$ phosphate buffer (PB; $\mathrm{pH}$ 7.4); and 3) $200 \mathrm{ml}$ of $2 \%$ paraformaldehyde in PB. Each brain was removed from the skull and blocked into $5 \mathrm{~mm}$ coronal blocks and post-fixed with $2 \%$ paraformaldehyde in PB for 30 min (Milner et al., 2001). The block was cut into $40 \mu \mathrm{m}$ thick sections on a vibrating microtome (Vibratome; Leica, Wein, Austria), and the sections were collected in PB and treated with $1 \%$ sodium borohydride in PB for 30 min prior to immunocytochemical labeling.

The tissue was processed for the immunocytochemical localization of total pLIMK according to the avidin-biotin complex (ABC) procedure (Milner et al., 2001). Briefly, sets of sections were incubated in (1) $0.5 \%$ bovine serum albumin (BSA) in $0.1 \mathrm{M}$ Tris-saline (TS), pH 7.6, for $30 \mathrm{~min}$; (2) anti-pLIMK antiserum (diluted $1: 1000$ ) in $0.1 \%$ BSA in TS for 1 day at room temperature and 1 day at $4{ }^{\circ} \mathrm{C}$; (3) biotinylated goat anti-rabbit IgG (1:400; Jackson Laboratories, Bar Harbor, ME, USA) for $30 \mathrm{~min}$; (4) peroxidase-avidin complex (Vector Laboratories, Burlingame, CA, USA) for $30 \mathrm{~min}$; and (5) diaminobenzidine (Aldrich, Milwaukee, WI, USA) and $\mathrm{H}_{2} \mathrm{O}_{2}$ for 6 min. Control sections were processed in the absence of pLIMK antiserum.

Sections prepared for LM were mounted on acid-cleaned slides previously coated with $1 \%$ gelatin, air-dried, dehydrated, and coverslipped with DPX (Aldrich). The final LM preparations were examined and photographed with a Micropublisher digital camera (Q Imaging, Barnaby, British Columbia, Canada) attached to a Nikon 80i light microscope (Nikon, Melville, NY, USA).

Sections prepared for EM were embedded in EMbed 812 (Electron Microscopy Sciences, Fort Washington, PA, USA [EMS]) between two sheets of Aclar plastic (Milner et al., 2001). Sections through the CA1 region of the dorsal hippocampus were glued onto Epon chucks and trimmed to a $1 \mathrm{~mm}$ trapezoid. Ultrathin sections (75 $\mathrm{nm}$ thick) through the hippocampal CA1 region were cut on a Leica Ultracut ultratome, collected into copper grids and counterstained with 5\% uranyl acetate and Reynolds lead citrate as described previously (Milner et al., 2001). Final preparations were analyzed on a FEI Tecnai Biotwin electron microscope equipped with a digital camera (Advanced Microscopy Techniques, software version 3.2). pLIMK immunoreactive (IR) profiles were classified according to the nomenclature of Peters et al. (1991). Perikarya were identified by the presence of a nucleus. Dendrites contained regular microtubular arrays and were usually postsynaptic to axon terminal profiles. Terminal profiles had minimal diameters greater than $0.2 \mu \mathrm{m}$, contained numerous small synaptic vesicles and often contacted other neuronal profiles. Final photomicrographs were generated from digital images with a Macintosh computer 8500/120 using Adobe Photoshop 7.0 (Adobe Systems) and Quark X-Press 4.1.

\section{Post-embedding EM}

Animals. Eleven young (3-4 month; $225 \mathrm{~g}$ ) and 12 aged (23-24 month; $350 \mathrm{~g}$ ) female Sprague-Dawley rats from Harlan (Indianapolis, IN, USA) were used in the post-embedding immunogold electron microscopic analysis. Animals were housed in a temperature-controlled room (12-h light/dark cycle; lights on at 07:00 h). Food and water were available ad libitum. All experiments were conducted in accordance with Guidelines for the Care and Use of Experimental Animals, by using protocols approved by the Institutional Animal Care and Use Committee at Mount Sinai School of Medicine. 
Ovariectomy (OVX) and estrogen replacement. Bilateral OVX was performed under isoflurane anesthesia. After 7 days, a Silastic capsule (capsule dimensions: inner diameter $1.96 \mathrm{~mm}$; outer diameter $3.18 \mathrm{~mm}$ ) filled with either $17 \beta$-estradiol $(E)(10 \%$ in cholesterol) or vehicle (Veh, i.e. cholesterol) was implanted s.c. under anesthesia. Young animals received an implant that was $1 \mathrm{~cm}$ in length and aged animals received an implant that was $2 \mathrm{~cm}$ in length. Different implant lengths were used for animals of different ages to account for differences in body weight (Lauber et al., 1990; Funabashi et al., 1998), which, as demonstrated previously (Adams et al., 2001b), results in comparable physiological $\mathrm{E}$ levels in young and aged rats. The animals were treated with either $E$ or Veh for 2 days prior to tissue harvest. The post-embedding immunogold analysis was done on ovariectomized female rats, half of which were treated with $E$ and half with Veh. These same animals have been used to demonstrate the effects of aging and $E$ on synapse number (Adams et al., 2001c), synaptic ER $\alpha$-IR (Adams et al., 2002) and synaptic $N$-methyl-Daspartate (NMDA) receptor profiles (Adams et al., 2004)

All young rats were cycling and all aged rats were acyclic (i.e. constant estrous or diestrus), as was demonstrated previously in our laboratory in a different cohort of animals (Adams et al., 2001a). In addition, the effectiveness of Veh and $E$ regimen after OVX was determined by examining the uterus from each animal. In both young and aged rats treated with Veh, uteri were very small and atrophied, and those that received $E$ displayed uterine hypertrophy. We previously used a similar $E$ replacement paradigm in a longer-term OVX in aged animals and observed a similar uterine response and the circulating $E$ levels were within a physiological range (Adams et al., 2001b).

Tissue preparation. Animals were anesthetized with $30 \%$ chloral hydrate (i.p.) and perfused transcardially with $2 \%$ dextran in $0.1 \mathrm{M} \mathrm{PB}(\mathrm{pH} 7.4,50 \mathrm{ml} / \mathrm{min}$ ) for $1 \mathrm{~min}$, followed by $4 \%$ paraformaldehyde and $0.125 \%$ glutaraldehyde in PB for $10-15$ min (Adams et al., 2001c, 2002, 2004). The carcasses were examined to confirm complete removal of both ovaries and the presence of the implant. No animals in the present study had obvious pituitary tumors, incomplete removal of ovaries, or lack of implant. The brains were removed and postfixed overnight. Two blocks from the dorsal hippocampus (1 $\mathrm{mm}$ thick) were randomly selected from each animal and processed for postembedding immunogold.

Post-embedding immunogold electron microscopic labeling. Freeze substitution and low-temperature embedding of the specimens were performed as described previously (van Lookeren Campagne et al., 1991; Adams et al., 2002; Janssen et al., 2005). Slices were cryoprotected by immersion in increasing concentrations of glycerol in PB $(10,20$, and $30 \%)$ and were plunged rapidly in liquid propane cooled by liquid nitrogen $\left(-190^{\circ} \mathrm{C}\right)$ in a Universal Cryofixation System KF80 (Reichert-Jung, Vienna). The samples were immersed in $1.5 \%$ uranyl acetate (for en bloc fixation) in anhydrous methanol $\left(-90^{\circ} \mathrm{C}, 24 \mathrm{~h}\right)$ in a cryosubstitution Automatic Freeze-Substitution System unit (Leica). The temperature was increased in steps of $4{ }^{\circ} \mathrm{C} / \mathrm{h}$ from -90 to $-45{ }^{\circ} \mathrm{C}$. The samples were washed with anhydrous methanol and infiltrated with Lowicryl $\mathrm{HM} 20$ resin (EMS) at $-45{ }^{\circ} \mathrm{C}$ with a progressive increase in the ratio of resin to methanol for $1 \mathrm{~h}$ each, followed by pure Lowicryl (overnight). Polymerization was performed with UV light $(360 \mathrm{~nm})$ at $-45{ }^{\circ} \mathrm{C}$ for $48 \mathrm{~h}$, followed by $24 \mathrm{~h}$ at room temperature.

An area (150-200 $\mu \mathrm{m}$ from the cell bodies) in the dorsal region (approximately level 32 , Swanson) of stratum radiatum of CA1 was sectioned. Pairs of ultrathin sections $(75 \mathrm{~nm}$ in thickness) were cut by diamond knife on a Reichert-Jung ultramicrotome and mounted on a nickel mesh grid for immunogold analysis. The mesh grids with ultrathin sections for the immunolabeling studies were treated with a saturated solution of sodium hydroxide in absolute ethanol, rinsed, and incubated in the following solutions at room temperature: $0.1 \%$ sodium borohydride and $50 \mathrm{mM}$ glycine, and then in TS containing $2 \%$ human serum albumin (HSA). The same anti-pLIMK antibody (Cell Signaling Technologies) was used for the post-embedding electron microscopic analysis as was used in the preembedding EM analysis. All the experiments and measurements were conducted for total pLIMK, and all results were given for total pLIMK distribution.

For single immunolabeling, grids containing sections were incubated with primary rabbit polyclonal anti-pLIMK antibody (Cell Signaling Technologies [diluted 1:10]) overnight. The next day, grids containing sections were washed, and incubated in secondary gold-tagged goat-anti-rabbit $\operatorname{lgG} \mathrm{F}\left(\mathrm{ab}^{\prime}\right)_{2}(10 \mathrm{~nm})$ antibody (EMS [diluted 1:30]) in TBS (2\% HSA and polyethyleneglycol 20,000; $5 \mathrm{mg} / 1 \mathrm{ml}$ ). Sections were washed and dried, counterstained with $1 \%$ uranyl acetate and Reynolds lead citrate. The sections were analyzed on a JEOL 1200EX electron microscope (JEOL, Tokyo, Japan). Images were captured by using the Advantage charge-coupled device camera (Advanced Microscopy Techniques, Danvers, MA, USA).

Controls for specificity of labeling by anti-pLIMK antibody. Controls for specificity of labeling by the anti-pLIMK antibody were performed. Control grids with thin sections were processed exactly as grids for the antibody experiments up to the step of applying the primary antibody. 1) To control for the nonspecific adsorption of the protein-gold complex of the secondary antibody to the tissue section, the anti-pLIMK antibody was omitted. Omitting the primary antibody resulted in no gold particles at all on the tissue sections. 2) To control for nonspecific adsorption of the protein-gold complex of the secondary antibody to the tissue section, the secondary antibody was replaced with normal nonrabbit secondary antibody, at the same protein concentration as well. Only occasional gold particles were seen, and they appeared to be randomly distributed.

Blocking experiments and preincubation of anti-pLIMK antibody. In order to verify the specificity of the interaction between the antibody and its antigen at the surface of the tissue section, the anti-pLIMK antibody was preadsorbed with the fusion protein provided by Cell Signaling Technologies. Again, only occasional gold particles were seen, and they appeared to be randomly distributed. Meanwhile, a run with the primary antibody omitted and a run in the presence of primary antibody were performed during this control experiment at the same time. For the controls, the tissue was examined and the results recorded by an investigator blind to the experimental conditions.

Also, another anti-pLIMK antibody (Chemicon, Temecula, CA, USA) was used in this study to determine if the distribution pattern was similar to the anti-pLIMK antibody from Cell Signaling Technologies. This polyclonal antibody was generated in the rabbit, and the immunogen was KHL-conjugated, a synthetic peptide corresponding to either amino acids $630-647$ or $616-633$ of alternatively spliced forms of human LIMK-1 containing an amino terminal lysine. The immunizing sequence has $17 / 18$ identical amino acids in mouse and rats.

Double immunolabeling for anti-pLIMK antibody. For double-labeling experiments, a mouse monoclonal $\operatorname{lgG}_{2 a}$ antibody directed against a protein corresponding to amino acids 495-595 mapping at the carboxy terminus of ER- $\alpha$ of human origin (C-311; Santa Cruz Biotechnology, Inc., CA, USA), was used. It detects ER- $\alpha$ of mouse, rat, bovine and human origin by immunocytochemistry. This antibody has been used extensively by different investigators before (Chen et al., 1997; Driscoll et al., 1998; Knoblauch and Garabedian, 1999; Rogatsky et al., 1999; Zink et al., 2002). During the double-labeling experiments, simultaneous incubation of mouse monoclonal anti-ER- $\alpha$ (C-311; Santa Cruz Biotechnology, Inc. [diluted 1:10]) with rabbit polyclonal anti- 
pLIMK antibody (Cell Signaling Technologies [diluted 1:10]) was performed overnight, followed with goat-anti-mouse $\operatorname{lgG} F\left(a b^{\prime}\right)_{2}$ $15 \mathrm{~nm}$ gold-tagged antibody (EMS [diluted 1:30]) in TBS. After washing with TBS, sections were incubated with goat-anti-rabbit $\operatorname{lgG~} \mathrm{F}\left(\mathrm{ab}^{\prime}\right)_{2} 5 \mathrm{~nm}$ gold-tagged antibody (EMS [diluted 1:30]). Control immunohistochemistry runs for anti-ER- $\alpha$ antibody were performed without primary antibody and with irrelevant secondary antibody.

Analysis of percentage of pLIMK-labeled synapses. An analysis was performed of the percentage of synaptic profiles with pLIMK-IR. Any synapse that contained at least two gold particles per postsynaptic profile (i.e. in the synaptic cleft, PSD, or spine head) was considered positive for pLIMK-IR (Adams et al., 2002, 2004). Photographs were taken at a magnification of 7500 . At least 150 synapses were counted for each animal and the analyses were performed by an experimenter blind to age and $E$ treatment.

Sub-synaptic distribution of pLIMK. The immunogold particle density and distribution in various compartments were analyzed using SYNBIN software (Adams et al., 2001c, 2002, 2004), that is based on principles regarding proximity to membranes articulated by Ruud and Blackstad (1999). Accordingly, the position of each gold particle was determined as it relates to the postsynaptic and presynaptic membrane structures. The program analyzes the resulting data map and objectively assigns each gold particle to a given bin, with bin sizes and targeted synaptic domains formed prospectively. The bin sizes are established on the basis of the lateral resolution of the EM techniques and to optimally separate synaptic and nonsynaptic pools of receptors. This process allows for determination of a precise gold particle/bin density that is an accurate reflection of gold particle distribution and density in different compartments of the synaptic complex (Adams et al., 2002, 2004). Gold particle analysis was done on an average of 35 randomly chosen spines per animal at a magnification of 40,000. Any synapse that contained at least two gold particles per postsynaptic profile (i.e. in the synaptic cleft, PSD, or spine head) was included for the synaptic bin analysis. Synapses cut obliquely that lacked clear visualization and delineation of classic synaptic structures such as presynaptic and postsynaptic membranes, a synaptic cleft, and PSD were excluded from the quantitative analysis.

For the present analysis, $30 \mathrm{~nm}$ was chosen for the bin width to assure high resolution yet comfortably accommodate the theoretical limit of resolution of the immunogold technique (i.e. $25 \mathrm{~nm}$ ). As shown in Fig. 3, the following zones were defined for each synapse (Adams et al., 2001c, 2002, 2004): a bin 0-30 nm from the inner leaflet of the postsynaptic membrane (Bin 1); a zone $30-60 \mathrm{~nm}$ from the postsynaptic membrane (Bin 2); two side bins that were $15 \mathrm{~nm}$ lateral to Bin 1 (Bin 3); a cytoplasmic bin that included gold particles $>60 \mathrm{~nm}$ from the postsynaptic membrane (Bin 4); the synaptic cleft (Bin 5); (5) a presynaptic zone extending $0-30 \mathrm{~nm}$ from the inner border of the presynaptic membrane (Bin 6); another presynaptic zone extending 30-60 nm from the inner border of the presynaptic membrane (Bin 7); and a presynaptic bin that included gold particles $>60 \mathrm{~nm}$ from the presynaptic membrane (Bin 8). With such a design, gold particles in the 0-30 nm PSD bin are unquestionably synaptic in location, whereas all other postsynaptic bins may include particles representing nonsynaptic pools of pLIMK. In addition, the lateral bins were used to establish a "buffer zone" at the lateral edge of the synapse to account for gold particles at the edge (i.e. within $15 \mathrm{~nm}$ ) that might be labeling proteins associated with the PSD. For the statistical analysis, since the gold particles in Bin 3 (i.e. $15 \mathrm{~nm}$ lateral to both of the postsynaptic bins) are the extension of Bin $1(0-30 \mathrm{~nm}$ from the inner leaflet of the postsynaptic membrane), they were added to Bin 1 .

Statistical analysis. Statistical analyses were performed using StatView 5.0 (Abacus Concepts, Inc., Berkeley, CA, USA). The coefficients of variation ( $\mathrm{CV}=$ standard deviation/mean) and coefficient error (CE) were calculated. Potential group differences in percentage of labeled synapses and number of gold particles per synaptic compartment between young ovariectomized Vehand estrogen-treated animals, as well as aged ovariectomized Veh- and estrogen-treated rats, were evaluated by unpaired $t$ tests. The grouped data were compared with analysis of variance (ANOVA), and multiple comparisons were made by Tukey-Kramer adjustments. Significance was set at $P<0.05$. All values are given as means \pm S.E.M. All images were captured and further processed using Adobe Photoshop 9.0. Only minor adjustments of brightness and contrast and sharpening were made, which in no case altered the appearance of the original material.

\section{RESULTS}

\section{In the hippocampal formation, pLIMK-IR is prominent in CA1 pyramidal neurons}

By LM, pLIMK-IR was detected predominantly in the CA1 subfield of the hippocampal formation whereas less was observed in the CA3 subfield and the dentate gyrus (Fig. 1A). Within the CA1 region, intense pLIMK labeling was associated with pyramidal cell somata in the pyramidal cell layer and with dendritic processes in stratum
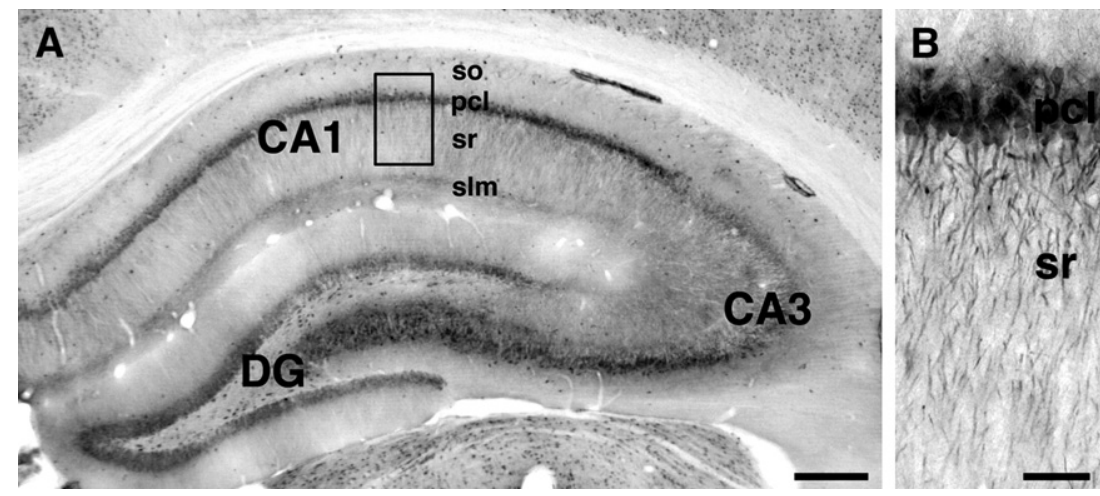

Fig. 1. Light microscopic localization of pLIMK-IR in the dorsal hippocampal formation. pLIMK-IR was detected in all major subfields, with the lowest intensity in CA3. Scattered pLIMK-labeled interneurons were found in striatum oriens (so) of CA1 and CA3. Dense, diffuse pLIMK-IR was seen in the stratum lacunosum moleculare $(\mathrm{slm})$ of CA1 (A). Within the CA1 region, strong pLIMK-labeling was associated with cell bodies in the pyramidal cell layer $(\mathrm{pcl})$ and processes in stratum radiatum (B). Scale bars $=2 \mathrm{~mm}$ A; B, $200 \mu \mathrm{m}$. (B) Higher magnification of boxed region in A. 


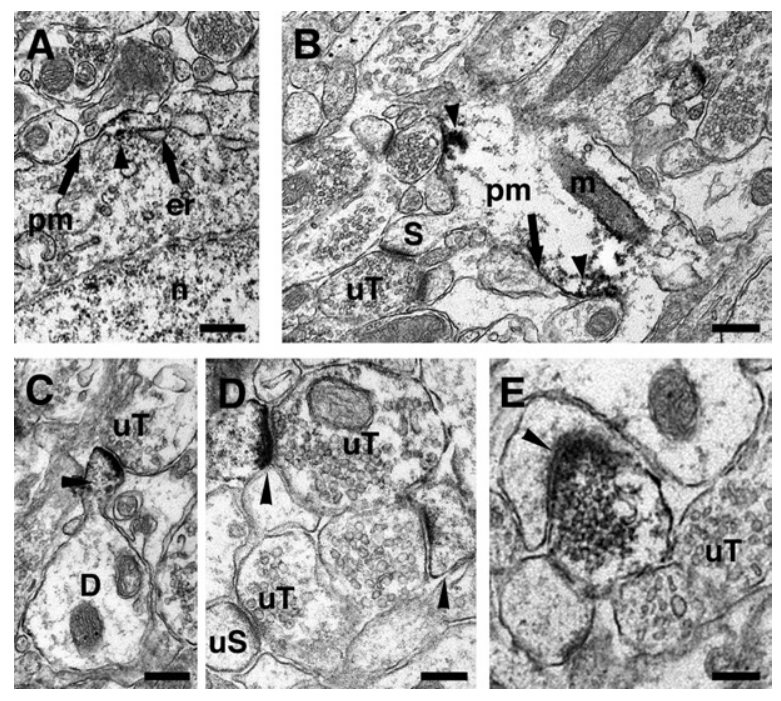

Fig. 2. Pre-embedding electron microscopic localization of pLIMK-IR in the hippocampal CA1 region. (A) Within perikarya, pLIMK-peroxidase IR (arrowhead) was associated (arrows) with the plasma membrane $(\mathrm{pm})$ and endoplasmic reticulum (er). (B) Within a dendritic shaft, pLIMK-IR (arrowheads) was affiliated with the plasma membranes and mitochondrion $(\mathrm{m})$ of a dendritic shaft. An unlabeled dendritic spine $(S)$ is shown emanating from the dendritic shaft in contact with an unlabeled terminal (UT). C. pLIMK-IR (arrowhead) is found in a dendritic $S$ originating from a dendritic shaft (D) and contacted by an UT. (D) Two pLIMK-labeled (arrowheads) dendritic $S$ are found in the same field. For comparison unlabeled spine (US) is shown. E. A pLIMK-labeled terminal forms an asymmetric synapse (arrowhead) with an unlabeled dendritic S. $n$, Nucleus; $S$, spine. Scale bar $=300 \mathrm{~nm}$ for A-C, D-E: $100 \mathrm{~nm}$.

radiatum (Fig. 1B). Scattered pLIMK-IR interneurons were found in striatum oriens of CA1 and CA3, and dentate hilus. Dense, diffuse pLIMK-IR was seen in the stratum lacunosum-moleculare of CA1 (Fig. 1A). No IR was detected in tissue processed in the absence of primary antibody.

Qualitative analysis of the CA1 region in immunoperoxidase-labeled sections from tissue prepared for pre-embedding EM showed that pLIMK-IR was prominently associated with perikarya, but not the nuclei, of neurons in the pyramidal cell layer and with dendritic profiles in stratum radiatum (Fig. 2A). Within perikarya and dendrites, pLIMK-IR was affiliated with plasma membranes and mitochondria (Fig. 2B). pLIMK-labeling was found throughout the dendritic shaft (Fig. 2B) and in select spines (Fig. 2C, D). In some cases, spines emanating from pLIMK-labeled dendritic shafts appeared unlabeled (Fig. 2B). Rarely, pLIMK-IR also was found in axon terminals (0.5-1.0 $\mu \mathrm{m}$ in diameter) that contained numerous small synaptic vesicles (Fig. 2E). Occasionally, pre- and post-synaptic profiles within the same spine-synapse complex contained pLIMK-IR. pLIMK-labeled terminals formed almost exclusively asymmetric synapses on dendritic spines.

\section{pLIMK-IR is most prominent in the PSD in all groups}

Since our pre-embedding EM analysis revealed that pLIMK-IR is found in pre- and post-synaptic profiles, postembedding immunocytochemistry was performed to quantitatively examine the subcellular distribution of pLIMK at higher resolution (see Fig. 3 for distribution scheme). For this, we analyzed the distribution of pLIMK within axospinous synapses in young and aged female rats that were ovariectomized and treated with either estrogen or Veh to determine whether pLIMK is affected by circulating estrogen or age (Adams et al., 2002).

In agreement with our pre-embedding electron microscopic findings, post-embedding immunogold labeling showed that pLIMK-IR is present in all compartments of the synapse, including the non-synaptic component of the spine (Fig. 4A-C). Within a given group, the synaptic distribution of pLIMK-IR was more pronounced in the postsynaptic compartment with prominent labeling within the PSD, particularly the central portion (Fig. 4C). Within the post-synaptic region, the distribution of pLIMK gold particles was non-uniform in all groups. The highest number of pLIMK gold particles was within 0-30 nm of the membrane associated with PSD with approximately $40 \%$ as many gold particles in the adjacent bin 30-60 nm from the membrane associated with PSD as well as a significant representation of gold particles in the non-synaptic core of the spine (Fig. 5).

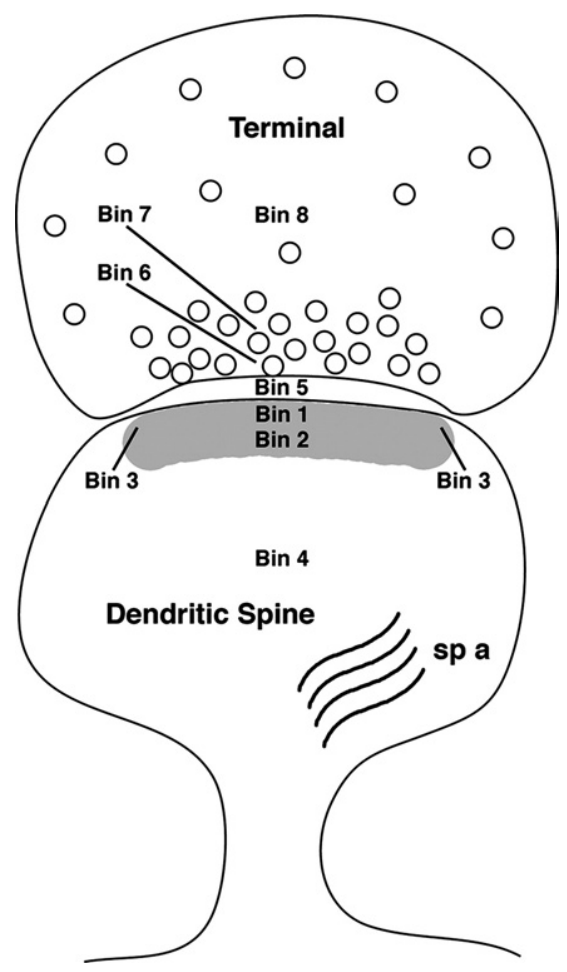

Fig. 3. Schematic diagram of bins in the terminal spine complex that were examined with the post-embedding EM analysis. Two major postsynaptic bins were designated as Bin $1(0-30 \mathrm{~nm}$ from the inner leaflet of the postsynaptic membrane), and Bin $2(30-60 \mathrm{~nm}$ from the postsynaptic membrane). Bins 3 are side bins, $15 \mathrm{~nm}$ lateral to Bins 1 and 2. Bin 4 is a post-synaptic cytoplasmic bin that includes gold particles $60 \mathrm{~nm}$ from the postsynaptic membrane. Bin 5 is the synaptic cleft. Two major presynaptic zones were designated as Bin 6 (0$30 \mathrm{~nm}$ from the inner border of the presynaptic membrane) and Bin 7 $(30-60 \mathrm{~nm}$ from the presynaptic membrane). Bin 8 is a presynaptic bin that includes gold particles $60 \mathrm{~nm}$ from the presynaptic membrane. $s p$ a, Spine apparatus. 

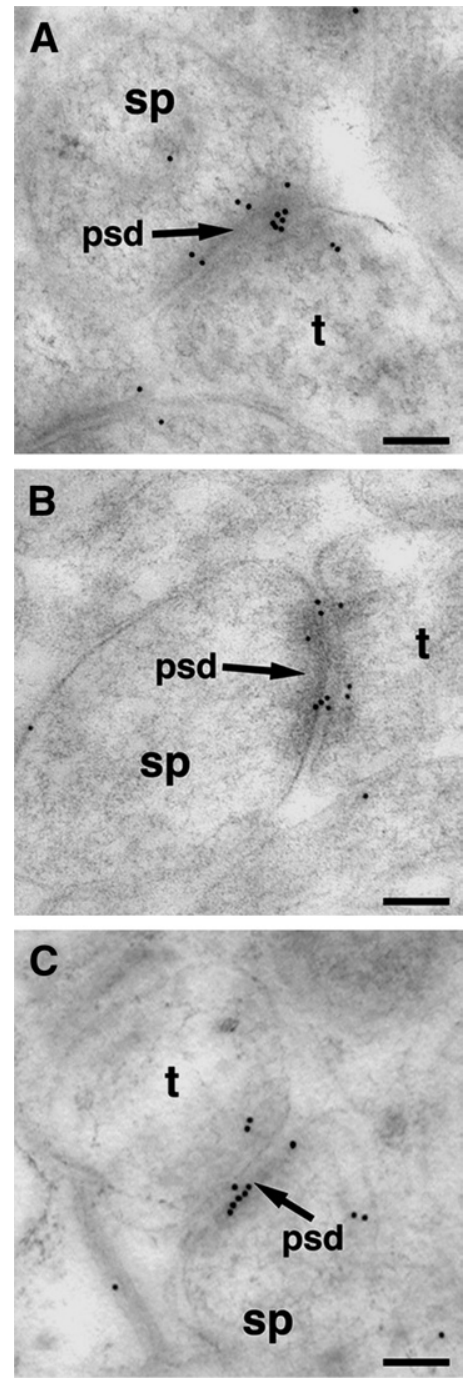

Fig. 4. Post-embedding electron microscopic distribution of pLIMK-IR within axospinous synapses in striatum radiatum of the hippocampus $\mathrm{CA} 1$ region. In young OVX $+E$ female rat $(\mathrm{A})$ and in young $\mathrm{OVX}+\mathrm{Veh}$ female rat (B), pLIMK immunogold particles (10 $\mathrm{nm}$ in diameter) are seen in pre-synaptic (Bins 6, 7 and 8), cleft (Bin 5) and post-synaptic (Bins 1, 2, and 4) compartments of the synapse. pLIMK immunogold particles are affiliated with the PSD and in the post-synaptic pool (Bins 1,2 , and 4) as well as pre-synaptically (Bins 6, 7 and 8) in an aged $\mathrm{OVX}+\mathrm{E}$ female rat (C). $t$, Terminal; $s p$, spine; $E$, E treated; Veh, Veh treated. Scale bars $=0.1 \mu \mathrm{m}$ for $\mathrm{A}-\mathrm{C}$.

\section{Estrogen treatment and aging primarily affect the post-synaptic distribution of pLIMK-IR}

With respect to sub-synaptic compartments, E increased gold particle counts in the non-synaptic core of the spine, e.g. cytoplasmic pool (Bin 4), in young animals $(P=0.05)$ (Fig. $5 \mathrm{~A} /$ Table 1 ), but not in aged animals (Fig. 5B/Table 1). The age-by-treatment interaction showed a similar trend for Bin 4 (>60 nm from the postsynaptic membrane) but failed to reach a $P$ value of $0.05(P=0.08)$. The multiple comparisons analysis by Tukey-Kramer adjustments shows that the main contribution to the group affect, although it was marginal, on Bin 4 (>60 nm from the postsynaptic membrane) was the difference between groups young $\mathrm{OVX}+\mathrm{E}$ and young
OVX+Veh (i.e. treatment effect, given young animals; $P=0.06$ ) (Fig. 5A/Table 1). Even though neither age nor $\mathrm{E}$ treatment alone was found to have any effect on the distribution of pLIMK gold particles in the synaptic compartments, the age-by-treatment interaction was significant for Bin 2 (30-60 nm from the postsynaptic membrane) $(P=0.05)$ (Fig. $5 /$ Table 1). Statistically, no significant differences between groups, or with aging or $E$ treatment were detected in the 0-30 nm bin post-synaptically (Bin 1), cleft (Bin 5), and 0-30 nm pre-synaptically (Bin 6) (Fig. 5/Table 1).

\section{Estrogen increases, while aging decreases, pLIMK labeling associated with synapses}

The percentage of pLIMK-IR associated with synapses increased with $E$ treatment in young animals, and decreased with age; however, there was no effect of $E$ treatment in the aged female rats (Fig. 6). The multiple comparisons analysis using the Tukey-Kramer adjustments revealed that, young OVX+E- and young OVX+Vehtreated groups are significantly different (i.e. treatment effect, for young animals; $P=0.02$ ) and that aged $\mathrm{OVX}+\mathrm{E}$ and young $\mathrm{OVX}+\mathrm{E}$ also are significantly different from each other (i.e. age effect, given $\mathrm{E} ; P=0.04$ ). The overall group effect is highly significant $(P=0.005)$. The main contribution to the significant overall group effect is the difference between the young $\mathrm{OVX}+\mathrm{E}$ and all three other groups, regardless of treatment or age. Age-by-treatment interaction is significant as well $(P=0.004)$. Young OVX $+\mathrm{E}$ has $30 \%$ more pLIMK immunogold particles associated with synapses then young OVX + Veh $(P<0.02)$, and $43 \%$ more labeled synapses then aged OVX+E $(P<0.002)$.

\section{Distribution of pLIMK-IR and ER- $\alpha$-IR showed a similarity within axospinous synapses}

Post-embedding electron microscopic distribution of pLIMK-IR showed a resemblance to the ER- $\alpha$-IR within axospinous profiles in striatum radiatum of the CA1 region. This colocalization was observed in the pre-synaptic compartment of the synapse (Bin 8) (Fig. 7A), in the PSD (Bins 1, 2, and 5) (Fig. 7B), $15 \mathrm{~nm}$ lateral to the PSD (Bin 3), and the nonsynaptic central portion of the spine (Bin 4) (Fig. 7C).

\section{DISCUSSION}

Several important observations emerged from this ultrastructural analysis of pLIMK distribution in CA1 and its potential alteration by estrogen status and/or age. First, pLIMK-IR is present primarily in postsynaptic profiles in CA1, with far less presynaptic profiles labeled. The postsynaptic localization is most predominant within PSDs, particularly within $30 \mathrm{~nm}$ of the membrane, but is also present in the non-synaptic core of the spine. This distribution is quite similar to that demonstrated for ER- $\alpha$ in axospinous synapses in rat CA1 (Milner et al., 2001; Adams et al., 2002), though pLIMK is more prominent in the PSD than ER- $\alpha$. In addition, the predominant localization within the PSD is very similar to cofilin (Racz and Weinberg, 2006) providing significant opportunity for binding to cofilin within the synapse, though pLIMK is more abundant 

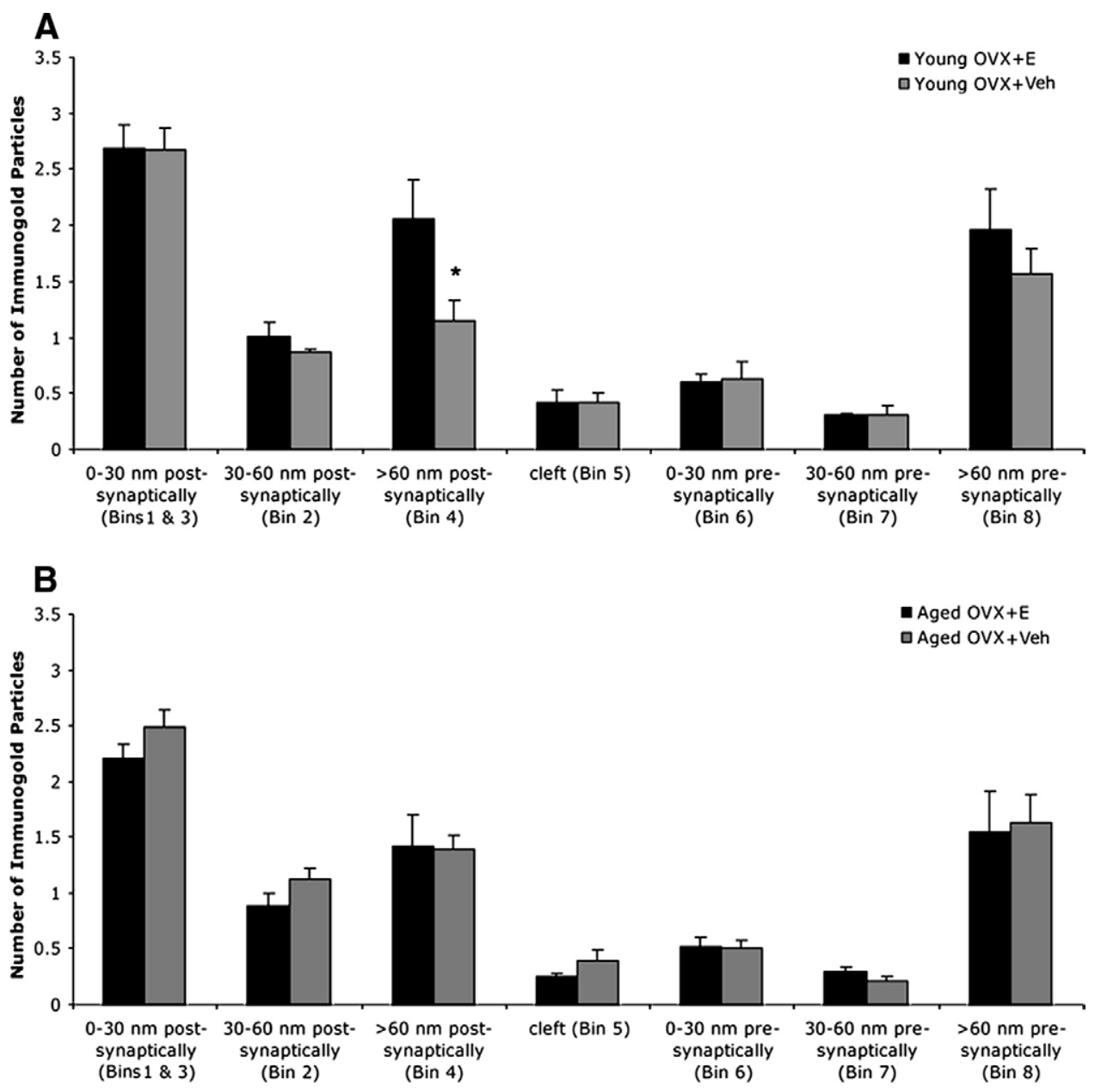

Fig. 5. Comparison of the immunogold particles in pre-synaptic, cleft and post-synaptic compartments of the synapse in four groups; A, ovariectomized $(O V X)$ and $E$ - or Veh-treated young female rats; B, OVX and E- or Veh-treated aged female rats. Within each group, pLIMK is mostly detected in the post-synaptic compartment. Within the post-synaptic region, the distribution of gold particles is non-uniform: highest within 0-30 nm (Bin 1) and $15 \mathrm{~nm}$ lateral (Bin 3) of the membrane associated with PSD, intermediate in the non-synaptic compartment of the spine (Bin 4) and the least in 30-60 nm of the membrane associated with PSD (Bin 2). $\left(^{*}\right)$ In A indicates E increased gold particle counts in the non-synaptic portion of the spine $(>60 \mathrm{~nm})$ in young animals $(P=0.05)$, but not in aged animals. The age-by-treatment interaction showed a similar trend in the non-synaptic portion of the spine $(>60 \mathrm{~nm})$, but failed to reach a $P$ value of $0.05(P=0.08)$. The values are given as mean \pm S.E.M.

in the interior core of the spine than is cofilin (Racz and Weinberg, 2006). Second, estrogen treatment in ovariectomized young animals increases the percentage of pLIMK-labeled synapses, yet there is an age-associated decrease in pLIMK-IR that is unresponsive to $E$ treatment. This result is consistent with two previous observations from these same animals: 1) estrogen induces an increase in synapse number in young animals, but fails to affect the

Table 1. Levels of immunogold pLIMK in all synaptic compartments in young and aged estrogen- and Veh-treated rats

\begin{tabular}{|c|c|c|c|c|}
\hline Bin designation & Young OVX+E $(N=5)$ & Young OVX+Veh $(N=6)$ & Aged OVX+E $(N=6)$ & Aged OVX + Veh $(N=6)$ \\
\hline Postsynaptic 0-30 nm & $2.68 \pm 0.22$ & $2.68 \pm 0.19$ & $2.21 \pm 0.12$ & $2.49 \pm 0.16$ \\
\hline Postsynaptic 30-60 nm & $1.01 \pm 0.12$ & $0.86 \pm 0.04$ & $0.89 \pm 0.11$ & $1.13 \pm 0.09$ \\
\hline Postsynaptic >60 nm & $2.06 \pm 0.34$ & $1.14 \pm 0.18^{*}$ & $1.42 \pm 0.27$ & $1.39 \pm 0.12$ \\
\hline Cleft & $0.42 \pm 0.12$ & $0.42 \pm 0.09$ & $0.26 \pm 0.03$ & $0.39 \pm 0.10$ \\
\hline Presynaptic 0-30 nm & $0.60 \pm 0.08$ & $0.63 \pm 0.16$ & $0.52 \pm 0.08$ & $0.50 \pm 0.08$ \\
\hline Presynaptic $30-60 \mathrm{~nm}$ & $0.30 \pm 0.02$ & $0.30 \pm 0.08$ & $0.29 \pm 0.04$ & $0.21 \pm 0.04$ \\
\hline Presynaptic $>60 \mathrm{~nm}$ & $1.96 \pm 0.36$ & $1.57 \pm 0.21$ & $1.54 \pm 0.37$ & $1.63 \pm 0.26$ \\
\hline
\end{tabular}

The numbers are the average number of pLIMK-immunolabeled gold particles per synapse in different compartments. $\left({ }^{*}\right)$ Indicates; $E$ increased number of pLIMK immunoreactive gold particles in the non-synaptic portion of the spine (>60 nm) in young animals $(P=0.05)$, but similar effect was not observed in aged animals. The age-by-treatment interaction showed a similar response in the non-synaptic portion of the spine $(>60 \mathrm{~nm})$ but failed to reach a $P$ value of $0.05(P=0.08)$. The numbers are given as mean \pm S.E.M. $E$, E treated; Veh, Veh treated. 


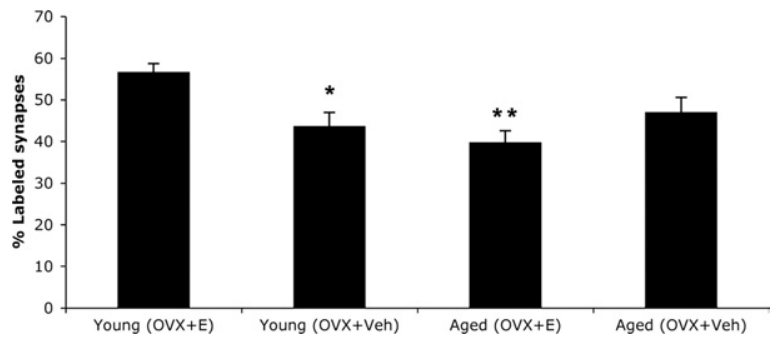

Fig. 6. Percentage of synapses containing pLIMK-IR in young and aged rats. The graphs illustrate that the percentage of immunogold labeled synapses increases $\left({ }^{*}, 30 \%\right)$ with $\mathrm{E}$ treatment $(P=0.02)$ in young animals, and decreases $\left({ }^{* *}, 43 \%\right)$ with age in the presence of $E$ $(P=0.04)$; however, there is no effect of $\mathrm{E}$ treatment between aged $\mathrm{OVX}+\mathrm{Veh}$ and aged $\mathrm{OVX}+\mathrm{E}$ groups $(P=0.38)$. Aged $\mathrm{OVX}+\mathrm{E}$ group and young $\mathrm{OVX}+\mathrm{E}$ group are significantly different from each other (i.e. age effect, given $\mathrm{E} ; P=0.04$ ), and that groups young $\mathrm{OVX}+\mathrm{E}$ and young $\mathrm{OVX}+\mathrm{Veh}$ are also significantly different (i.e. treatment effect, for young animals; $P=0.02$ ). $N=5$ for young $\mathrm{OVX}+\mathrm{E}$ and is 6 for other treatment groups. The values are given as mean \pm S.E.M. E, E-treated; Veh, Veh-treated.

age-associated decrease in synapses in CA1 (Adams et al., 2001c); and 2) synaptic $E R \alpha$-IR is responsive to $E$ in young animals but unresponsive in aged animals where $50 \%$ fewer CA 1 synapses even contain ER- $\alpha$-IR. These data support the hypothesis that the age-related inability of $E$ treatment to increase synapse number in CA1 may be due to alterations in synaptic ER- $\alpha / p L I M K / c o f i l i n$ interactions that regulate actin dynamics required for formation of additional spines and synapses in response to $\mathrm{E}$.

\section{Methodological considerations}

For electron microscopic comparisons of pLIMK-IR, the pre-embedding and post-embedding immunocytochemical methods were used. Pre-embedding EM provides distinct subcellular localization of reaction product while maintaining morphological preservation (Leranth and Pickel, 1989) and is more suitable than post-embedding methods for localization of IR at extra-synaptic sites and for determining regional distributions (Lujan et al., 1996). However, the localization of synaptic pLIMK-IR using the pre-embedding immunogold method is less suitable for quantification than is the post-embedding immunogold electron microscopic method, particularly for quantification in different compartments within the synapse/spine complex. Because of these different strengths, we used both methods to localize pLIMK in hippocampus.

Spatial convergence of ER- $\alpha, p L I M K$, and cofilin. The distribution within the spine of pLIMK is strikingly similar to $\mathrm{ER} \alpha$ in that it is predominant within the PSD yet occasionally present presynaptically and reasonably abundant within the core of the spine, as is ER- $\alpha$. This overlapping distribution provides ample opportunity for local ER$\alpha /$ pLIMK interaction within CA1 synapses. In addition, while cofilin is poorly represented in the core of the spine, it is preferentially localized within the PSD of CA1 spines (Racz and Weinberg, 2006), providing for direct spatial overlap of cofilin and pLIMK within the PSD. Since pLIMK impacts actin depolymerization through phosphorylation of cofilin, their spatial overlap within the PSD provides an important opportunity for pLIMK to facilitate translation of synaptic activity into altered spine/synapse morphology, as has been proposed for cofilin (Racz and Weinberg, 2006). Cofilin is directly linked to spine alterations resulting from LTD (Zhou et al., 2004) and LTP (Fedulov et al., 2007) and pLIMK has been directly linked to altered spine morphology (Meng et al., 2002, 2003). Our data taken together with a similar analysis of cofilin (Racz and Weinberg, 2006) provide direct ultrastructural evidence that both proteins are present in CA1 PSDs and thus capable of the direct
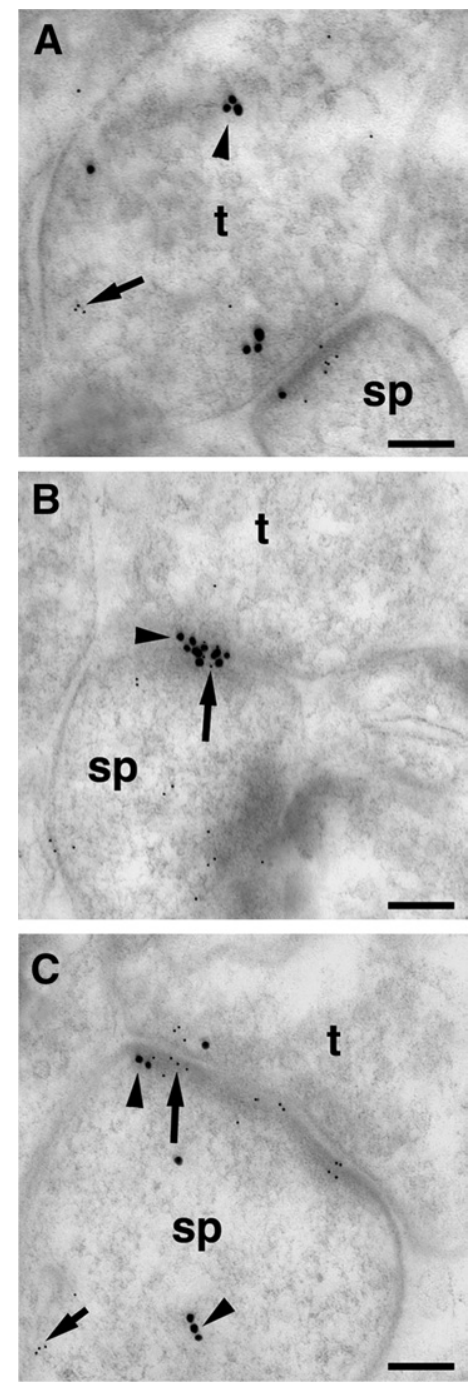

Fig. 7. Post-embedding electron microscopic distribution of pLIMK-IR relative to the ER- $\alpha-I R$ within axospinous profiles in striatum radiatum of the CA1 region in a young OVX+E female rat. (A) In the synapse, immunoreactivities for pLIMK ( $5 \mathrm{~nm}$ particles; arrow) and ER- $\alpha$ (15 nm particles, arrowhead) in pre-synaptic compartment of the synapse (Bin 8) were observed. (B) In a synapse, immunoreactivities for pLIMK (5 nm particles; arrow) and ER- $\alpha$ (15 nm particles, arrowhead) are seen in the PSD of the synapse (Bins 1, 2, 5). (C) In the post-synaptic compartment of the synapse, immunoreactivities for pLIMK (5 nm particles; arrow) and ER- $\alpha$ (15 nm particles, arrowhead) are seen $15 \mathrm{~nm}$ lateral of the post- synaptic density (Bin 3 ) and in the post-synaptic compartment (Bin 4). $t$, Terminal; $s p$, spine. Scale bars $=0.1 \mu \mathrm{m}$ for $\mathrm{A}-\mathrm{C}$. 
interaction within the synapse that would facilitate such linkage.

\section{Decreased levels of ER- $\alpha$ and pLIMK immunoreactivities correlate with the absence of estrogen-induced spine formation in aging hippocampus}

The age-associated decrease in pLIMK-IR associated with the synapse demonstrated here and coincident with agerelated decrease in $\mathrm{ER} \alpha$ likely contributes to E's inability to impact spine formation and synapse number in aged female rats. Several lines of evidence suggest that pLIMK plays a critical role in dendritic spine morphogenesis. In LIMK - /- mice, the necks of the spines are significantly thicker while the head region is reduced in size, resulting in decreased spine area and length of the PSD (Meng et al., 2002). In addition to changes in spine morphology, growth cones of hippocampal neurons cultured from LIMK -/mice are smaller or absent compared with those cultured from LIMK + /+ control mice (Meng et al., 2002). Moreover decreased spatial learning and altered fear responses have been observed (Meng et al., 2002). If spine morphology and surface area are disrupted in the synapse, accumulation of the proper signaling molecules in the PSD would likely be affected by filament concentration in spines. High receptor concentration at the postsynaptic membrane, established by actin dependent spine morphology, is necessary for proper neuronal transmission (Sarmiere and Bamburg, 2002), and pLIMK plays a critical role in dendritic spine morphogenesis and brain function. It is essential for proper accumulation and distribution of actin filaments in the dendritic branches and spines and critical to establishment and/or maintenance of normal spine morphology in vivo and in culture (Meng et al., 2002).

Data presented here demonstrate that the presence of $E$ is associated with an increase in synaptic pLIMK levels in young female rats. This is consistent with biochemical and immunohistochemical studies directly demonstrating an E-induced increase in neuronal pLIMK mediated by ER- $\alpha$ (Yuen et al., 2004). In addition, both ER- $\alpha$ and LIMK have been associated with similar signal transduction pathways (Wang et al., 2002; Mazumdar and Kumar, 2003). However, by linking $E$ to increased synaptic pLIMK, this study offers support on a synaptic level for E stimulation of actin-dependent dendritic spine formation through pLIMK. Since E modulates pLIMK activity (Yuen et al., 2004) and pLIMK activity is known to be crucial to actin rearrangement (Edwards et al., 1999; Maekawa et al., 1999), then the absence of circulating $E$ in the aging brain would likely contribute to decreased signaling and actin rearrangement through this pathway, leading to a decline in synapse number and related cognitive effects. In addition to nuclear labeling in interneurons (Weiland et al., 1997), ER $\alpha$-IR is present within the pre-synaptic terminals and dendritic spines of CA1 pyramidal neurons (Milner et al., 2001). Moreover, ER- $\alpha$ redistributes at the synapse in response to $E$ in young animals, but does not in aged rats (Adams et al., 2002) Since the enhanced spine formation may be mediated by ER- $\alpha$ present at the synapse, then the age-related loss of synaptic ER- $\alpha$-IR would lead to compromised ER- $\alpha$ induced signal transduction. A similar decrease in synaptic pLIMK-IR suggests that ER- $\alpha / L I M K /$ cofilin/actin interactions are compromised with age, leading to a failure of E-induced synaptogenesis.

Acknowledgments-This study was supported by NIH-NIA grant P01-AG16765 (J.H.M.) and NS07080 (B.S.M. and T.A.M.).

\section{REFERENCES}

Adams MM, Fink SE, Janssen WG, Shah RA, Morrison JH (2004) Estrogen modulates synaptic $\mathrm{N}$-methyl-D-aspartate receptor subunit distribution in the aged hippocampus. J Comp Neurol 474:419-426.

Adams MM, Fink SE, Shah RA, Janssen WG, Hayashi S, Milner TA, McEwen BS, Morrison JH (2002) Estrogen and aging affect the subcellular distribution of estrogen receptor-alpha in the hippocampus of female rats. J Neurosci 22:3608-3614.

Adams MM, Morrison JH, Gore AC (2001a) N-methyl-D-aspartate receptor mRNA levels change during reproductive senescence in the hippocampus of female rats. Exp Neurol 170:171-179.

Adams MM, Oung T, Morrison JH, Gore AC (2001b) Length of postovariectomy interval and age, but not estrogen replacement, regulate N-methyl-D-aspartate receptor mRNA levels in the hippocampus of female rats. Exp Neurol 170:345-356.

Adams MM, Shah RA, Janssen WG, Morrison JH (2001c) Different modes of hippocampal plasticity in response to estrogen in young and aged female rats. Proc Natl Acad Sci U S A 98:8071-8076.

Arber S, Barbayannis FA, Hanser H, Schneider C, Stanyon CA, Bernard O, Caroni $P$ (1998) Regulation of actin dynamics through phosphorylation of cofilin by LIM-kinase. Nature 393:805-809.

Bamburg JR (1999) Proteins of the ADF/cofilin family: essential regulators of actin dynamics. Annu Rev Cell Dev Biol 15:185-230.

Carlier MF, Laurent V, Santolini J, Melki R, Didry D, Xia GX, Hong Y, Chua NH, Pantaloni D (1997) Actin depolymerizing factor (ADF/ cofilin) enhances the rate of filament turnover: implication in actinbased motility. J Cell Biol 136:1307-1322.

Carlisle HJ, Kennedy MB (2005) Spine architecture and synaptic plasticity. Trends Neurosci 28:182-187.

Chen H, Arbelle JE, Gacad MA, Allegretto EA, Adams JS (1997) Vitamin $D$ and gonadal steroid-resistant New World primate cells express an intracellular protein which competes with the estrogen receptor for binding to the estrogen response element. J Clin Invest 99:669-675.

Day M, Sung A, Logue S, Bowlby M, Arias R (2005) Beta estrogen receptor knockout (BERKO) mice present attenuated hippocampal CA1 long-term potentiation and related memory deficits in contextual fear conditioning. Behav Brain Res 164:128-131.

Driscoll MD, Sathya G, Muyan M, Klinge CM, Hilf R, Bambara RA (1998) Sequence requirements for estrogen receptor binding to estrogen response elements. J Biol Chem 273:29321-29330.

Edwards DC, Sanders LC, Bokoch GM, Gill GN (1999) Activation of LIM-kinase by Pak1 couples Rac/Cdc42 GTPase signalling to actin cytoskeletal dynamics. Nat Cell Biol 1:253-259.

Endo M, Ohashi K, Sasaki Y, Goshima Y, Niwa R, Uemura T, Mizuno K (2003) Control of growth cone motility and morphology by LIM kinase and slingshot via phosphorylation and dephosphorylation of cofilin. J Neurosci 23:2527-2537.

Fedulov V, Rex CS, Simmons DA, Palmer L, Gall CM, Lynch G (2007) Evidence that long-term potentiation occurs within individual hippocampal synapses during learning. J Neurosci 27:8031-8039.

Foletta VC, Lim MA, Soosairajah J, Kelly AP, Stanley EG, Shannon M, He W, Das S, Massague J, Bernard O (2003) Direct signaling by the BMP type II receptor via the cytoskeletal regulator LIMK1. J Cell Biol 162:1089-1098. 
Fukazawa Y, Saitoh Y, Ozawa F, Ohta Y, Mizuno K, Inokuchi K (2003) Hippocampal LTP is accompanied by enhanced F-actin content within the dendritic spine that is essential for late LTP maintenance in vivo. Neuron 38:447-460.

Funabashi T, Kleopoulos SP, Kimura F, Mobbs CV (1998) Changes in neurotensin mRNA by estrogen in the female rat preoptic area during aging: an in situ hybridization histochemistry study. Gen Comp Endocrinol 112:364-371.

Gould E, Woolley CS, Frankfurt M, McEwen BS (1990) Gonadal steroids regulate dendritic spine density in hippocampal pyramidal cells in adulthood. J Neurosci 10:1286-1291.

Janssen WG, Vissavaijhala P, Andrews G, Moran T, Hof PR, Morrison JH (2005) Cellular and synaptic distribution of NR2A and NR2B in macaque monkey and rat hippocampus as visualized with subunit-specific monoclonal antibodies. Exp Neurol 191 (Suppl 1):S28-S44.

Jelks KB, Wylie R, Floyd CL, McAllister AK, Wise P (2007) Estradiol targets synaptic proteins to induce glutamatergic synapse formation in cultured hippocampal neurons: critical role of estrogen receptor-alpha. J Neurosci 27:6903-6913.

Knoblauch R, Garabedian MJ (1999) Role for Hsp90-associated cochaperone p23 in estrogen receptor signal transduction. Mol Cell Biol 19:3748-3759.

Lauber AH, Romano GJ, Mobbs CV, Howells RD, Pfaff DW (1990) Estradiol induction of proenkephalin messenger RNA in hypothalamus: dose-response and relation to reproductive behavior in the female rat. Brain Res Mol Brain Res 8:47-54.

Leranth C, Pickel VM (1989) Electron microscopic preembedding doublelabeling methods. In: Neuroanatomical tract-tracing methods (Heimer L, Zaborszky L, eds), pp 129-172. New York: Plenum Press.

Lujan R, Nusser Z, Roberts JD, Shigemoto R, Somogyi P (1996) Perisynaptic location of metabotropic glutamate receptors mGluR1 and mGluR5 on dendrites and dendritic spines in the rat hippocampus. Eur J Neurosci 8:1488-1500.

Maekawa M, Ishizaki T, Boku S, Watanabe N, Fujita A, Iwamatsu A, Obinata T, Ohashi K, Mizuno K, Narumiya S (1999) Signaling from Rho to the actin cytoskeleton through protein kinases ROCK and LIM-kinase. Science 285:895-898.

Mazumdar A, Kumar R (2003) Estrogen regulation of Pak1 and FKHR pathways in breast cancer cells. FEBS Lett 535:6-10.

Meberg PJ (2000) Signal-regulated ADF/cofilin activity and growth cone motility. Mol Neurobiol 21:97-107.

Meng Y, Takahashi H, Meng J, Zhang Y, Lu G, Asrar S, Nakamura T, Jia Z (2004) Regulation of ADF/cofilin phosphorylation and synaptic function by LIM-kinase. Neuropharmacology 47:746-754.

Meng Y, Zhang Y, Tregoubov V, Falls DL, Jia Z (2003) Regulation of spine morphology and synaptic function by LIMK and the actin cytoskeleton. Rev Neurosci 14:233-240.

Meng Y, Zhang Y, Tregoubov V, Janus C, Cruz L, Jackson M, Lu WY, MacDonald JF, Wang JY, Falls DL, Jia Z (2002) Abnormal spine morphology and enhanced LTP in LIMK-1 knockout mice. Neuron 35:121-133.

Milner TA, Ayoola K, Drake CT, Herrick SP, Tabori NE, McEwen BS, Warrier S, Alves SE (2005) Ultrastructural localization of estrogen receptor beta immunoreactivity in the rat hippocampal formation. J Comp Neurol 491:81-95.

Milner TA, McEwen BS, Hayashi S, Li CJ, Reagan LP, Alves SE (2001) Ultrastructural evidence that hippocampal alpha estrogen receptors are located at extranuclear sites. J Comp Neurol 429:355-371.

Mukai $\mathrm{H}$, Tsurugizawa T, Murakami G, Kominami S, Ishii $\mathrm{H}$, OgiueIkeda M, Takata N, Tanabe N, Furukawa A, Hojo Y, Ooishi Y, Morrison JH, Janssen WG, Rose JA, Chambon P, Kato S, Izumi S, Yamazaki T, Kimoto T, Kawato S (2007) Rapid modulation of long-term depression and spinogenesis via synaptic estrogen receptors in hippocampal principal neurons. J Neurochem 100: 950-967.

Mukai H, Tsurugizawa T, Ogiue-lkeda M, Murakami G, Hojo Y, Ishii H, Kimoto T, Kawato S (2006) Local neurosteroid production in the hippocampus: influence on synaptic plasticity of memory. Neuroendocrinology 84:255-263.

Ohashi K, Hosoya T, Takahashi K, Hing H, Mizuno K (2000a) A Drosophila homolog of LIM-kinase phosphorylates cofilin and induces actin cytoskeletal reorganization. Biochem Biophys Res Commun 276:1178-1185.

Ohashi K, Nagata K, Maekawa M, Ishizaki T, Narumiya S, Mizuno K (2000b) Rho-associated kinase ROCK activates LIM-kinase 1 by phosphorylation at threonine 508 within the activation loop. J Biol Chem 275:3577-3582.

Okamoto K, Nagai T, Miyawaki A, Hayashi Y (2004) Rapid and persistent modulation of actin dynamics regulates postsynaptic reorganization underlying bidirectional plasticity. Nat Neurosci 7: $1104-1112$.

Okano I, Hiraoka J, Otera H, Nunoue K, Ohashi K, Iwashita S, Hirai M, Mizuno K (1995) Identification and characterization of a novel family of serine/threonine kinases containing two N-terminal LIM motifs. J Biol Chem 270:31321-31330.

Peters A, Palay SL, Webster H (1991) The fine structure of the nervous system: neurons and their supporting cells. 3rd edition. New York: Oxford University Press Inc.

Racz B, Weinberg RJ (2006) Spatial organization of cofilin in dendritic spines. Neuroscience 138:447-456.

Rogatsky I, Trowbridge JM, Garabedian MJ (1999) Potentiation of human estrogen receptor alpha transcriptional activation through phosphorylation of serines 104 and 106 by the cyclin A-CDK2 complex. J Biol Chem 274:22296-22302.

Rosenblatt J, Agnew BJ, Abe H, Bamburg JR, Mitchison TJ (1997) Xenopus actin depolymerizing factor/cofilin (XAC) is responsible for the turnover of actin filaments in Listeria monocytogenes tails. J Cell Biol 136:1323-1332.

Ruud HK, Blackstad TW (1999) PALIREL, a computer program for analyzing particle-to-membrane relations, with emphasis on electron micrographs of immunocytochemical preparations and gold labeled molecules. Comput Biomed Res 32:93-122.

Sarmiere PD, Bamburg JR (2002) Head, neck, and spines: a role for LIMK-1 in the hippocampus. Neuron 35:3-5.

Sarmiere PD, Bamburg JR (2004) Regulation of the neuronal actin cytoskeleton by ADF/cofilin. J Neurobiol 58:103-117.

Sumi T, Matsumoto K, Nakamura T (2001) Specific activation of LIM kinase 2 via phosphorylation of threonine 505 by ROCK, a Rhodependent protein kinase. J Biol Chem 276:670-676.

Szymczak S, Kalita K, Jaworski J, Mioduszewska B, Savonenko A, Markowska A, Merchenthaler I, Kaczmarek L (2006) Increased estrogen receptor beta expression correlates with decreased spine formation in the rat hippocampus. Hippocampus 16:453-463.

van Lookeren Campagne M, Oestreicher AB, van der Krift TP, Gispen WH, Verkleij AJ (1991) Freeze-substitution and Lowicryl HM20 embedding of fixed rat brain: suitability for immunogold ultrastructural localization of neural antigens. J Histochem Cytochem 39:1267-1279.

Vardouli L, Moustakas A, Stournaras C (2005) LIM-kinase 2 and cofilin phosphorylation mediate actin cytoskeleton reorganization induced by transforming growth factor-beta. J Biol Chem 280: $11448-11457$.

Wang RA, Mazumdar A, Vadlamudi RK, Kumar R (2002) P21-activated kinase-1 phosphorylates and transactivates estrogen receptor-alpha and promotes hyperplasia in mammary epithelium. EMBO J 21:5437-5447.

Wang Y, Shibasaki F, Mizuno K (2005) Calcium signal-induced cofilin dephosphorylation is mediated by slingshot via calcineurin. J Biol Chem 280:12683-12689.

Weiland NG, Orikasa C, Hayashi S, McEwen BS (1997) Distribution and hormone regulation of estrogen receptor immunoreactive cells in the hippocampus of male and female rats. J Comp Neurol 388:603-612.

Wen Z, Han L, Bamburg JR, Shim S, Ming GL, Zheng JQ (2007) BMP gradients steer nerve growth cones by a balancing act of LIM 
kinase and slingshot phosphatase on ADF/cofilin. J Cell Biol 178:107-119.

Woolley CS, McEwen BS (1992) Estradiol mediates fluctuation in hippocampal synapse density during the estrous cycle in the adult rat. J Neurosci 12:2549-2554.

Woolley CS, Wenzel HJ, Schwartzkroin PA (1996) Estradiol increases the frequency of multiple synapse boutons in the hippocampal CA1 region of the adult female rat. J Comp Neurol 373:108-117.

Yang N, Higuchi O, Ohashi K, Nagata K, Wada A, Kangawa K, Nishida E, Mizuno K (1998) Cofilin phosphorylation by LIMkinase 1 and its role in Rac-mediated actin reorganization. Nature 393:809-812.
Yuen GS, Akama KT, McEwen BS (2004) Estrogen regulation of actin dynamics: mechanism for dendritic spine remodeling. Soc Neurosci 72.5, Online.

Zhou Q, Homma KJ, Poo MM (2004) Shrinkage of dendritic spines associated with long-term depression of hippocampal synapses. Neuron 44:749-757.

Zhou Q, Xiao M, Nicoll RA (2001) Contribution of cytoskeleton to the internalization of AMPA receptors. Proc Natl Acad Sci U S A 98:1261-1266.

Zink D, Mayr C, Janz C, Wiesmuller L (2002) Association of p53 and $\mathrm{MSH} 2$ with recombinative repair complexes during $\mathrm{S}$ phase. Oncogene 21:4788-4800. 